\title{
Improved Local Lattice Approach for Coulombic Simulations
}

\author{
A. Duncan, ${ }^{1}$ R.D. Sedgewick, ${ }^{2}$ and R.D. Coalson ${ }^{2}$ \\ ${ }^{1}$ Department of Physics and Astronomy \\ University of Pittsburgh, Pittsburgh, PA 15260 \\ ${ }^{2}$ Department of Chemistry \\ University of Pittsburgh, Pittsburgh, PA 15260
}

\begin{abstract}
An improved approach to the simulation of strongly fluctuating Coulomb gases, based on a local lattice technique introduced by Maggs and Rossetto [1], is described and then tested in a problem of biophysical interest. The low acceptance rates for charged particle moves in regimes of physical interest are increased to a serviceable level by use of a coupled particle-field update procedure in the new method. Sensitivity of the results to lattice discretization effects is also studied using asymmetric lattices.
\end{abstract}

\section{INTRODUCTION}

The simulation of systems containing a large number of mobile charged entities, in which long range electrostatic forces play a central dynamical role, is of critical importance in modern chemical physics and biophysics. In many cases, the computational load is dominated by the evaluation of the electrostatic energy of the system, where the long-range character of the Coulomb interaction greatly complicates the development of efficient algorithms that scale with system size in a way that permits study of systems of biophysical interest. Developments in supercomputing technology aimed at large-scale biophysical simulations, such as the IBM BlueGene project [2], where massively parallel assemblies of processor nodes are coupled via a three-dimensional toroidal topology, suggest that algorithms based on a local energy functional will be much more efficiently executed on the next generation of high-end computing platforms than those involving long-range nonlocal effects.

Recently, Maggs and collaborators [1] have suggested an ingenious procedure for removing the nonlocal (longranged) Coulomb term in equilibrium simulations of Coulomb gases. By using a completely local Hamiltonian for a system of mobile charged particles interacting with the electrostatic field, one avoids the unpleasant scaling characteristics of conventional Coulomb gas simulations. Unfortunately (as pointed out by these authors themselves $3 \mid$ ), the algorithm they propose runs into serious acceptance problems in regions of physical interest (basically, for strongly fluctuating systems). In this paper, we study the origin of these acceptance problems and propose an improved algorithm that allows useful simulations of strongly fluctuating systems in which mean-field (or Poisson-Boltzmann) methods break down.

In Section 2 we briefly review the original technique of Maggs et al, and explain the origin of the acceptance difficulty for charged particle moves. In Section 3 we explain the modified update procedure designed to cure, or at least ameliorate, the acceptance problem for particle moves. In brief, the crucial point is to implement a coupled particle-field update in which the electrostatic field is allowed to readjust itself in tandem with charged particle moves in response to the changed electrostatic environment. In Section 4, the improved procedure is tested in detail on a system that has been extensively studied in the literature [4, 5, 6, 7]: the osmotic pressure of charged plates (or membranes) separated by an ionic fluid. Finally, in Section 5 we briefly summarize our conclusions.

\section{LOCAL LATTICE HAMILTONIANS FOR COULOMB GAS PROBLEMS}

The difficulties incurred by the nonlocal nature of the Coulomb interaction in realistic simulations of large systems (for example, for large biomolecular systems) are well known: the computational cost increases as the square of the number of charged constituents, and although various techniques (Ewald summation, fast Fourier transforms etc. 8]) can be employed to improve this scaling, the resulting complications in the algorithm often mean that the computation of the electrostatic energy still consumes essentially all of the computational effort, greatly limiting the size of the systems and (in the case of molecular dynamics simulations) the time frames over which the simulations can be extended. These techniques also have difficulties modeling a non-uniform dielectric constant, which is an important feature of many biophysical systems [9, 10, 11] as the dielectric constant in proteins is $\sim 2-8$ while the dielectric constant of water is $\sim 80$. In the case of systems at equilibrium, it has been known for some time [4, 12, 13, 14] that the nonlocal Coulomb interaction can be replaced by a completely local interaction via a Hubbard-Stratonovich transformation, yielding a path integral formalism that connects naturally with the Poisson-Boltzmann mean-field theory. Unfortunately, for strongly fluctuating systems perturbation theory (saddle-point expansions) breaks down in this approach, and a direct numerical simulation is obstructed by a severe sign problem.

Recently, Maggs and collaborators [1] have proposed an alternative, purely local approach to the simulation of charged condensed systems. They exploit the fact that the nonlocality of the Coulomb interaction is a con- 
sequence of a particular choice of gauge for describing the electromagnetic field, whereas the physically relevant quantity -the electrostatic energy of the system- must clearly be a gauge-invariant object. They propose that the electromagnetic field be simulated in terms of gaugeinvariant objects (specifically, the electric field), represented on a discrete spatial lattice. In this respect, the method proposed is essentially the same as that employed for over 20 years by elementary particle theorists attacking the problem of strong interactions with the technique of lattice quantum chromodynamics. For a review see Ref. 15. The main distinction here is that the gauge theory involved is the abelian one of Maxwellian electrodynamics, magnetic effects are not relevant, and the formulation used is a noncompact one (i.e. the electric field variables take unbounded values).

Let us briefly recall the salient points of the formalism of Maggs et al 1]. The canonical partition function for a set of mobile charges $e_{i}$ at locations $\vec{r}_{i}$ at inverse temperature $\beta$ in a medium of dielectric constant $\epsilon$ may be written

$Z=\int \prod_{i=1}^{N} d \vec{r}_{i} \mathcal{D} \vec{E}(\vec{r}) \prod_{\vec{r}} \delta\left(\vec{\nabla} \cdot \vec{E}-\frac{4 \pi}{\epsilon} \rho(\vec{r})\right) e^{-\frac{\beta \epsilon}{8 \pi} \int d \vec{r} \vec{E}(\vec{r})^{2}}$

where the charge density $\rho(\vec{r})$ is shorthand for

$$
\rho(\vec{r}) \equiv \sum_{i} e_{i} \delta\left(\vec{r}-\vec{r}_{i}\right)
$$

The delta function constraint in Eq. 11 enforces Gauss' Law, so that the electric fields integrated over correspond to the particle locations specified through the density function $\rho$. The formulation is manifestly local, as both the energy functional and Gauss' Law constraint are so. There is no requirement that the electric fields integrated over be irrotational, and in fact they are not; as shown by Maggs et al. 1], the transverse part of the electric field simply decouples from the particle sector and contributes an irrelevant overall prefactor to $Z$. Because this formalism is local, it is easily extended to model physical systems with a non-uniform dielectric constant [16].

The functional integral over electric field in Eq. 11 can be given a precise definition by introducing a spatial cubical lattice, which we shall for the time being take to be a grid of $L^{3}$ points, with lattice spacing $a$ (in all directions: the modifications needed in case of an asymmetric lattice are discussed below) and periodic wrap-around boundary conditions in all three spatial directions. The charges $e_{i}, i=1, . . N$ are assumed to be integer multiples of a basic unit of charge, $e_{i}=z_{i} e, z_{i}$ integer, and reside on the sites of the lattice. The component $E_{\mu}(\vec{n})$ of electric field in direction $\mu$ at lattice site $\vec{n}$ is associated with a real-valued field $E_{l}$ on the oriented link $l$ from $\vec{n}$ to $\vec{n}+\hat{\mu}$. Discretizing the 3 -dimensional integral for the electrostatic energy in the obvious way, we find

$$
H \equiv \frac{\epsilon}{8 \pi} \int d \vec{r} \vec{E}(\vec{r})^{2} \rightarrow \frac{a^{3} \epsilon}{8 \pi} \sum_{l} E_{l}^{2}
$$

The implementation of the simulation is simplified by introducing dimensionless variables to the greatest extent possible, so we define $\hat{E}_{l} \equiv \frac{\epsilon a^{2}}{4 \pi e} E_{l}$ and a rescaled inverse temperature $\hat{\beta} \equiv \frac{4 \pi e^{2} \beta}{\epsilon a}$, in terms of which the energy becomes

$$
H=\frac{\hat{\beta}}{2} \sum_{l} \hat{E}_{l}^{2}
$$

while the Gauss' Law constraint takes the simple form

$$
\sum_{l} \hat{E}_{l}=z_{i}
$$

for the sum of outgoing link fields from any site containing a charged particle of charge $z_{i} e$.

The simulation of the system defined by the energy function in Eq. 4 and the constraint in Eq. 5 can in principle be accomplished by the following algorithm:

1. Pick starting lattice locations (possibly randomly) for the $N$ particles of charge $z_{i}, i=1, . . N$. Then solve Gauss' Law for these fixed charge locations to obtain a starting configuration of electric link field variables satisfying the Gauss constraint. This can easily be done by standard numerical relaxation methods [17].

2. Update the electric fields by shifting all link variables along a complete set of independent closed paths by constant shifts, using either Metropolis or heat bath procedures to accept/reject proposed shifts. The simplest version of this is simply to consider all plaquettes (unit squares) on the lattice, shifting the 4 link fields ordered around the plaquette by the same random amount $\alpha$, the range of $\alpha$ set so that there is a reasonable acceptance rate for the move. Such a shift clearly maintains the Gauss' Law constraint.

3. Update particle locations by visiting in turn every site $\vec{n}$ containing a charged particle of charge $z_{i}$. A particle move to the neighboring site $\vec{n}+\hat{\mu}$ in a random direction $\mu$ is then considered, where the particle move is accompanied with a shift of the electric field $E_{l}$ on the link $l=(\vec{n} \rightarrow \vec{n}+\hat{\mu})$

$$
\hat{E}_{l} \rightarrow \hat{E}_{l}-z_{i}
$$

in order to maintain the constraint in Eq. 5. Here also one can employ either Metropolis or heat-bath accept/reject procedures.

The inclusion of additional force fields, for example soft or hard exclusion potentials modeling a finite size for the particles, is, in principle, completely straightforward in this framework. When particles are packed closely together, or the potential changes rapidly over the scale of a lattice spacing, then it is important to verify that 
the observed phenomena are not distorted by lattice discretization effects. It is useful to be able to study the effects of lattice discretization in such situations by introducing asymmetric lattices, in which the lattice spacing in the various directions differs. As a specific example, consider a situation in which we may desire a finer discretization in the $\mathrm{x}$-direction, relative to the $\mathrm{y}$ and $\mathrm{z}$ directions, $a_{x}<a_{y}=a_{z} \equiv a$. One readily verifies that with the choice of the dimensionless variables

$$
\hat{E}_{l} \equiv \begin{cases}\frac{\epsilon a^{2}}{4 \pi e} E_{l} & l \in L_{x} \\ \frac{\epsilon a a_{x}}{4 \pi e} E_{l} & l \in L_{y} \cup L_{z}\end{cases}
$$

where $L_{\alpha}$ is the set of links in the $\alpha$ direction and, as before,

$$
\hat{\beta}=\frac{4 \pi e^{2}}{\epsilon a} \beta
$$

the energy function becomes

$$
H=\frac{\hat{\beta}}{2}\left(\sum_{l \in L_{x}} \frac{a_{x}}{a} \hat{E}_{l}^{2}+\sum_{l \in L_{y}} \frac{a}{a_{x}} \hat{E}_{l}^{2}+\sum_{l \in L_{z}} \frac{a}{a_{x}} \hat{E}_{l}^{2}\right)
$$

while the Gauss' Law constraint retains its original form given in Eq. 5 .

Unfortunately, despite the appealing simplicity of the simulation procedure outlined above, in physically realistic situations involving strongly charged systems the method proves impractical, for reasons we now explain. The dimensionless inverse temperature variable $\hat{\beta}$ is typically large compared to unity (in the charged plate/membrane problem considered in Section 4, the value is 87.1 ), so that typical values for the electric field link variables are small compared to unity. On the other hand, executing a particle move across a link via Eq. 6 shifts the electric field variable on that link by an integer, and this generally leads to an unacceptable energy cost (on the order of $\hat{\beta}$ ). In the univalent case $\left(z_{i}= \pm 1\right.$ ), acceptance rates for particle moves are of the order of $10^{-4}$, while for divalent ions $\left(z_{i}= \pm 2\right)$ the acceptance rate is at best of order $10^{-6}$. Thus the unmodified procedure of Maggs et al. is clearly not a practical approach in situations approximating real biophysical systems. In the next section, we discuss a modified simulation algorithm in which this problem is ameliorated to an acceptable level.

\section{SOLVING THE PARTICLE MOVE PROBLEM: A COUPLED UPDATE PROCEDURE}

The problem of very inefficient particle moves mentioned in the preceding section needs to be resolved before the local Hamiltonian method can be applied fruitfully to realistic problems with strongly fluctuating Coulomb gases. Recall that the Hamiltonian, as a function of the electric field variables $E_{l}$ defined on the links

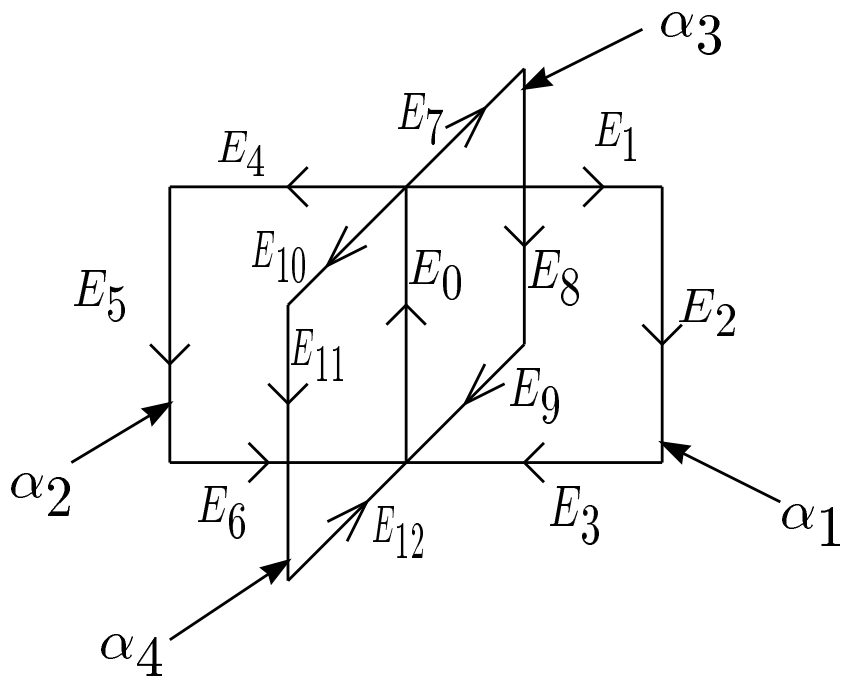

FIG. 1: Local field environment for coupled particle move updates

$l$ of the lattice takes the form

$$
\mathcal{H}=\frac{\hat{\beta}}{2} \sum_{l} \hat{E}_{l}^{2}
$$

where the dimensionless inverse-temperature variable, $\hat{\beta}$, is quite large for the systems that we are interested in studying (in the range of 50-100). As discussed above, the vast majority of particle moves with such a Hamiltonian have a high energy cost leading to an unacceptably low acceptance rate. In this section we will show that this problem can be substantially ameliorated- though not completely eliminated- by a coupled update procedure in which electric field values on all the plaquettes containing the link along which the particle move is attempted are simultaneously adjusted to reflect the changed electrical environment resulting from the particle move.

As an example of a simple procedure that can considerably improve the acceptance rate for particle moves, consider the situation illustrated in Fig. 11 Here we are considering the move of a unit charge particle from the beginning (bottom) site to the end (top) site of the central link associated with field variable $E_{0}$. In conjunction with the particle move, we consider simultaneous electric field updates corresponding to plaquette variable shifts $\alpha_{1}, \alpha_{2}, \alpha_{3}, \alpha_{4}$ on the four plaquettes containing the link $E_{0}$, as indicated in the figure. Such a combined move changes the energy associated with the illustrated region from

$$
\mathcal{H}_{\text {before }}=\frac{\hat{\beta}}{2} \sum_{l=0}^{12} \hat{E}_{l}^{2}
$$

to

$$
\mathcal{H}_{\text {after }}=\frac{\hat{\beta}}{2}\left\{\left(\hat{E}_{0}+\sum_{i=1}^{4} \alpha_{i}-1\right)^{2}\right.
$$




$$
\left.\begin{array}{l}
+\sum_{l=1}^{3}\left(\hat{E}_{l}+\alpha_{1}\right)^{2}+\sum_{l=4}^{6}\left(\hat{E}_{l}+\alpha_{2}\right)^{2} \\
+\sum_{l=7}^{9}\left(\hat{E}_{l}+\alpha_{3}\right)^{2}+\sum_{l=10}^{12}\left(\hat{E}_{l}+\alpha_{4}\right)^{2}
\end{array}\right\}
$$

In practice one finds that the electric field variables equilibrate to values which are small compared to unity: in the approximation where we simply set $\hat{E}_{l}=0$ in Equations 11$]$ and 12 the energy cost of the combined move becomes

$$
\Delta H=\frac{\hat{\beta}}{2}\left(\left(\sum_{i=1}^{4} \alpha_{i}-1\right)^{2}+3\left(\alpha_{1}^{2}+\alpha_{2}^{2}+\alpha_{3}^{2}+\alpha_{4}^{2}\right)\right)
$$

Minimizing Eq. 13 with respect to the $\alpha_{i}$, we find that the choice $\alpha_{i}=1 / 7$ gives the minimum energy cost

$$
\Delta H_{\min }=\frac{3}{7} \frac{\hat{\beta}}{2} \approx 0.43 \frac{\hat{\beta}}{2}
$$

as opposed to the cost $\hat{\beta} / 2$ if the particle move is unaccompanied by any readjustment of nearby link fields. As we shall see in the next section, if $\hat{\beta}$ is large, this is enough to increase the acceptance rate for particle moves to a level where configurations can be decorrelated at an acceptable rate. Thus, a quick and easily implementable improvement of the basic algorithm can be obtained by a Metropolis accept/reject step in which the choices for a particle move on a chosen link are (a) do nothing (to particle or fields), or (b) perform the combined update in which the particle is transferred to the end site of the link and the fields around the four intersecting plaquettes are shifted by $1 / 7$. It is clear that the energy cost can be further reduced by allowing readjustments of plaquettes adjacent to those depicted in Fig. 1 In particular, if

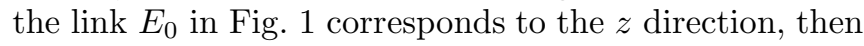
adding the remaining $x z$ and $y z$ plaquettes that contain the links $E_{2}, E_{5}, E_{8}$ and $E_{11}$, and performing the relevant minimization, one finds however that the reduction in energy cost is only about $10 \%$, with a considerable complication in the algorithm. In this paper we have chosen to implement only the simplest (most local) version of a coupled move-field update, corresponding to the situation in Fig. [1]

A more general procedure, in which a heat-bath update on the combined (particle move)+(field update) space provides a complete local decorrelation between adjacent Monte Carlo configurations can easily be derived as follows. We remind the reader that, in a heat-bath Monte Carlo update, parameters are introduced to characterize a subspace of the configuration space in the neighborhood of the starting configuration, the dependence of the full Boltzmann weight of the theory on these parameters is extracted (from the full Hamiltonian), and then new values for these parameters are then chosen (independent of the original configuration) on the basis of this Boltzmann weight. In the situation considered here, the parameter space consists of a single discrete particle move variable $m=0,1$ (with $m=0$ corresponding to no move, $m=1$ to a move along a specified link), and four continuous plaquette shift variables $\alpha_{i}, i=1, . .4$. For the indicated environment of the central link $E_{0}$ in Fig. 1 the relevant part of the Hamiltonian, as a function of $m$ and the continuous plaquette update variables $\alpha_{i}$, becomes

$$
\begin{aligned}
\mathcal{H}\left(m, \alpha_{i}\right) & =\frac{\hat{\beta}}{2}\left\{\left(\hat{E}_{0}+\sum_{i=1}^{4} \alpha_{i}-m z\right)^{2}\right. \\
& +\sum_{l=1}^{3}\left(\hat{E}_{l}+\alpha_{1}\right)^{2}+\sum_{l=4}^{6}\left(\hat{E}_{l}+\alpha_{2}\right)^{2} \\
& \left.+\sum_{l=7}^{9}\left(\hat{E}_{l}+\alpha_{3}\right)^{2}+\sum_{l=10}^{12}\left(\hat{E}_{l}+\alpha_{4}\right)^{2}\right\}
\end{aligned}
$$

where we have introduced a variable (integer) valence $z$ to take care of the case (needed in the simulations of Section 4) of multivalent ions. In order to implement a heat-bath procedure for this energy function, we need to generate values for the quintet $\left(m, \alpha_{1}, \alpha_{2}, \alpha_{3}, \alpha_{4}\right)$ distributed according to the Boltzmann weight $e^{-\mathcal{H}\left(m, \alpha_{i}\right)}$. Fortunately, a complete analytic solution to this problem can easily be derived. First, we note that the energy function in Eq. 15] can be reexpressed

$$
\begin{aligned}
\mathcal{H}\left(m, \alpha_{i}\right)= & \frac{\hat{\beta}}{2}\left\{\left(\hat{E}_{0}-m z\right)^{2}+\sum_{i, j} \alpha_{i} M_{i j} \alpha_{j}\right. \\
& \left.+2 \sum_{i} \lambda_{i} \alpha_{i}+\sum_{l=1}^{12} \hat{E}_{l}^{2}\right\}
\end{aligned}
$$

where

$$
\begin{aligned}
\lambda_{i} & \equiv P_{i}-m z \\
P_{1} & =\hat{E}_{0}+\hat{E}_{1}+\hat{E}_{2}+\hat{E}_{3} \\
P_{2} & =\hat{E}_{0}+\hat{E}_{4}+\hat{E}_{5}+\hat{E}_{6} \\
& \text { etc }
\end{aligned}
$$

i.e. the variables $P_{i}, i=1,2,3,4$ are just the plaquette fields obtained by summing the electric link variables around each of the four plaquettes containing the central link of Fig. 1] and the $4 \times 4$ matrix $M_{i j}$

$$
M_{i j}=3 \delta_{i j}+1
$$

is easily found to have inverse

$$
M_{i j}^{-1}=\frac{1}{3}\left(\delta_{i j}-\frac{1}{7}\right)
$$

Completing the square in Eq. 16] we find that $\mathcal{H}$ takes the form

$$
\mathcal{H}=\frac{\hat{\beta}}{2}\left\{\left(\hat{E}_{0}-m z\right)^{2}+\sum_{i j} \xi_{i} M_{i j} \xi_{j}\right.
$$




$$
\left.\begin{array}{rl} 
& -\sum_{i j} \lambda_{i} M_{i j}^{-1} \lambda_{j}+\sum_{l=1}^{12} \hat{E}_{l}^{2}
\end{array}\right\}
$$

The dependence of the local energy on the discrete move variable $m$ arises from the first and third terms in Eq. 23 and the corresponding Boltzmann weight determining the relative probability of a particle move $(m=1)$ versus no move $(m=0)$ is therefore

$$
\exp \left(\frac{\hat{\beta}}{2}\left(2 \hat{E}_{0} z-\frac{3}{7} z^{2}-\frac{2}{7} z \sum_{i} P_{i}\right) m\right)
$$

where we have used the fact that the move variable $m=$ 0,1 so that $m^{2}=m$. A heat bath update of the variable $m$ is therefore trivial to implement.

The continuous $\alpha_{i}$ variables can be generated easily from the Gaussian distribution of the $\xi_{i}$. The eigenvalues of $M_{i j}$ are easily found (they are $7,3,3,3$ ), as are the eigenvectors, whence we find that the contribution of the second term in Eq. 23$]$ to the Boltzmann weight can be rewritten

$$
\exp \left(-\frac{\hat{\beta}}{2}\left(7 \eta_{1}^{2}+3 \eta_{2}^{2}+3 \eta_{3}^{2}+3 \eta_{4}^{2}\right)\right)
$$

where

$$
\begin{aligned}
\eta_{1} & =\frac{1}{2}\left(\xi_{1}+\xi_{2}+\xi_{3}+\xi_{4}\right) \\
\eta_{2} & =\frac{1}{\sqrt{2}}\left(\xi_{1}-\xi_{2}\right) \\
\eta_{3} & =\frac{1}{\sqrt{2}}\left(\xi_{3}-\xi_{4}\right) \\
\eta_{4} & =\frac{1}{2}\left(\xi_{1}+\xi_{2}-\xi_{3}-\xi_{4}\right)
\end{aligned}
$$

The heat-bath procedure for the plaquette shifts $\alpha_{i}$ therefore amounts to generating the independent Gaussian distributed variables $\eta_{i}$ according to the weight (26), whence the $\alpha_{i}$ can be reconstructed via Equations 27.30 and

$$
\begin{aligned}
\alpha_{i} & =\xi_{i}-\sum_{j} M_{i j}^{-1} \lambda_{j} \\
& =\xi_{i}-\frac{1}{3} \lambda_{i}+\frac{1}{21} \sum_{j} \lambda_{j}
\end{aligned}
$$

To summarize, the algorithm for a coupled particle/field heat-bath update is implemented as follows:

1. Calculate the plaquette sums $P_{i}, i=1,2,3,4$ (Equations 18 20) for the four plaquettes interfacing the link along which we desire to move the particle.

2. Choose the move variable $m=0,1$ with weight given by Eq. 25]
3. Generate independent Gaussian variables $\eta_{i}, i=$ 1, 2,3, 4 according to Eq. 26]

4. Solve Equations 2730 for the $\xi_{i}, i=1,2,3,4$.

5. Compute $\lambda_{i}$ from Eq. 17 and use Eq. 32 to obtain the desired plaquette shifts $\alpha_{i}, i=1,2,3$, 4 , which are then used to update the electric fields $\hat{E}_{l}, l=$ $0, . .12$ as indicated in Eq. 15] (see Fig. 11).

6. If $m=1$ then move the particle across the considered link while updating the electric field on the link according to Eq. [6]

Recently the problem of low acceptance rates for particle moves was noted by Maggs et al. in Ref. 3 . They present an alternative solution to the problem where each charge, instead of residing on a single lattice site, is broken into pieces and resides on the lattice sites in an $n \times n \times n$ cube. In order to move a particle, all of the pieces of the particle must be moved in unison. They have shown that the inverse temperature that they are able to simulate efficiently grows like $n^{3}$ using this method. The advantage of this method is that it is effective at increasing the acceptance rate and that the size of the cube can be chosen to give the desired acceptance rate. The disadvantage of this method is that the charges are spread out so that, for systems that are sensitive to the spatial location of the charges, the lattice must be made finer by a factor of $n$ in every direction to obtain the same charge locality as the lattice with unbroken particles. Using the methods discussed in this work, the charges remain on a single lattice site so there are no difficulties arising from the breakup of the ions onto different lattice sites.

\section{APPLICATIONS: STRONGLY FLUCTUATING FIELDS BETWEEN CHARGED PLATES/MEMBRANES}

To test these algorithms on a strongly charged system where correlation effects play a major role, we have considered a system of charged conducting plates with ions between the plates. While the system is electrically neutral, there is an osmotic pressure between the plates that depends on the electrostatic interaction between the particles and on the correlations between the particles. This system has been extensively studied both theoretically $[\underline{4},[\underline{5}]$ and numerically [ㅁ, [7] and is known to be a strongly fluctuating system within the parameter ranges in which we are interested. A convenient criterion [7] for a strongly fluctuating system is that the Bjerrum length, $\ell_{B}$, times the square of the ion valence, $z$, be smaller then the Gouy-Chapman length, $\mu$. In the systems we are considering $z^{2} \ell_{B} / \mu$ is as large as 33 . We have considered both divalent and univalent ions, as in previous work it was seen that there is a repulsive pressure in the univalent case and an attractive pressure in the divalent case $4,6,6$, 7]. It is shown in Ref. [4 that the Poisson-Boltzmann calculations of the osmotic pressure 
in the divalent case break down and cannot even predict the sign of the osmotic pressure.

Our basic system consists of a 50x50x50 lattice with periodic boundary conditions in all three dimensions. Positive charges are free to move on two fixed plates separated in the $x$ direction which extend the entire extent of the lattice in the $y$ and $z$ directions, while the region between the plates contains mobile counterions ensuring overall neutrality. The periodicity in the $x$ direction is not critical, as quantities observed are insensitive to field fluctuations far outside the plates. We choose a lattice spacing of $1 \AA$, so that we can study plate separations in the range of interest. Using the dielectric constant of water $(\epsilon=80.0)$ and room temperature $(T=300 K)$ gives a dimensionless inverse temperature $\hat{\beta}=87.1$, too large to effectively simulate with simple particle moves that do not adjust the electric field on neighboring plaquettes. We placed 34 positive univalent charges on each of the plates to give a surface-charge density of $0.2176 \mathrm{Cm}^{-2}$, approximately that used in References 4, 6. These charges are allowed to move during the simulation, but are not allowed to leave the plate. To make the system electrically neutral, 68 negatively charged ions are placed between the plates in the univalent case, and 34 negatively charged ions in the divalent case. Two ions are forbidden from being on the same lattice site. The charges on the plates are initially randomly distributed on the plates, and the ions between the plates are initially distributed with half of the ions on the closest allowed plane to the right plate, and half on the closest allowed plane to the left plate. All runs are composed of 5,000 Monte Carlo equilibration steps followed by 20,000 measurement steps. Each Monte Carlo step is composed of a coupled Metropolis update of the electric field around each plaquette, $(200 \times$ Number of charges on the plates) attempted moves of a particle on the plate chosen at random, and $(20000 \times$ Number of charges in solution) attempted moves of a particle in solution. As pointed out by Maggs et al.[16], a global update of the electric field is also included to ensure rigorous ergodicity.

To investigate the importance of the mobility of the charges on the plates we have also performed a set of simulations with the positive charges on plates fixed at a random initial distribution. There were no qualitative differences between the results of these simulations and the results of the simulations with mobile ions on the plates that we present here.

To investigate the errors due to lattice effects, we have also studied an asymmetric lattice where the lattice spacing is a factor of two smaller in the dimension separating the plates. This is a $100 \times 50 \times 50$ asymmetric lattice with a lattice spacing of $0.5 \AA$ in the $x$ direction so that the total volume of the system remains constant. Two ions are again forbidden from being on the same lattice site. In the asymmetric case this corresponds to a different hard sphere interaction between the ions than in the symmetric lattice, but these differences are in practice unimportant, as the ions are so sparsely distributed that collision between ions are rare.

The ions between the plates will naturally accumulate on the planes of lattice sites close to the plates. As the electric potential changes rapidly in this region, the results of our simulation will depend on the details of the discretization in this region. As the discreteness of the lattice has the largest effect in the region close to the plates, we have chosen to forbid the ions from coming within $1 \AA$ of the plates. This will soften slightly the potential seen by the ions. On the symmetric lattice, we do not allow ions on the planes of lattice sites closest to the plates. On the asymmetric lattice, ions are not allowed on the 2 planes of lattice sites closest to the plates.

We are primarily interested in observing the osmotic pressure between the plates as we change the separation, both for the univalent and divalent ions in solution. As derived in Ref. 6 , the osmotic pressure can be calculated using the expression

$$
p_{\mathrm{Osm}}=k T C(0)+F_{x}^{A B} / \text { area },
$$

where $C(0)$ is the ion concentration at the mid-plane and $F_{x}^{A B}$ is the average electrostatic force between the left half of the system and the right half of the system. In the continuum, this force could be written as

$$
F_{x}^{A B}=\frac{1}{\epsilon} \sum_{m}^{A} \sum_{n}^{B} q_{n} q_{m} \Delta x_{m n} / r_{m n}^{3},
$$

where $A$ is the set of all charges to the left of the midplane, $B$ is the set of all charges to the right of the midplane, $\Delta x_{m n}$ is the separation between the charges in the $x$ direction, and $r_{m n}$ is the distance between the charges. In order to take into account lattice effects and correctly treat the periodic boundary conditions, we have calculated the force using the lattice coulomb force. This is done simply by replacing the continuum quantity $\Delta x_{m n} / r_{m n}^{3}$ in Eq. 34 by the corresponding lattice expression

$$
\frac{4 \pi}{L^{3}} \sum_{\vec{k} \neq 0} \frac{i \sin \left(2 \pi k_{x} / L\right) e^{2 \pi i \vec{k} \cdot \vec{r}_{m n}}}{4 \sum_{i=1}^{3} \sin ^{2}\left(\pi k_{i} / L\right)}
$$

where $k_{i}=0,1,2, \ldots . L-1$.

Although the osmotic pressure could be calculated by observing the change in free energy as the separation of the plates is changed, work in Ref. 6 has shown that the pressure calculated using Eq. 33. has fewer fluctuations than the pressure calculated using the free energy difference.

The pressure for univalent ions is shown in Fig. 2 for a range of plate separations. Results are shown from both the 50x50x50 lattice and the 100x50x50 lattice. The ions are moved using the coupled Metropolis update method described in Sec. IIII as the heat bath method is difficult to adapt to the asymmetric lattice. Figure 3 shows the first term of Eq. 33 the concentration of ions on the plane between the plates, for the same simulations. The 


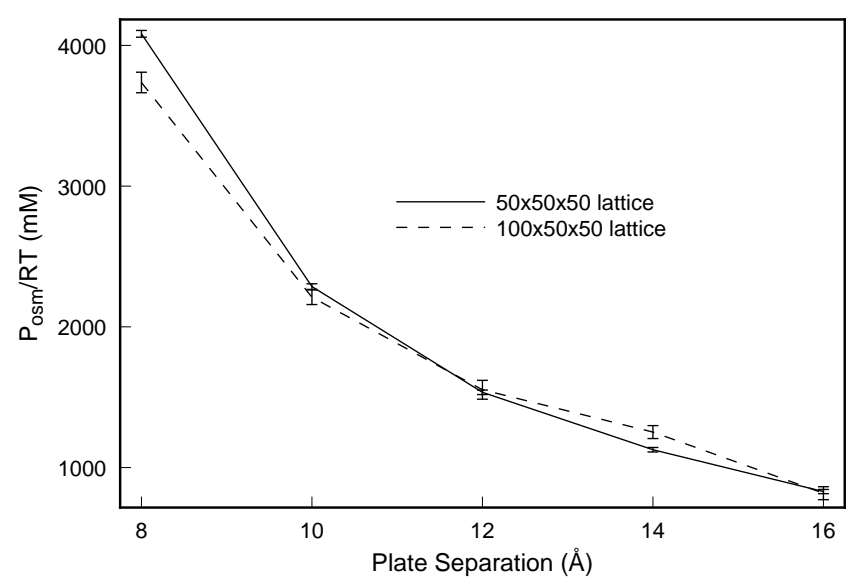

FIG. 2: Osmotic pressure from simulations of univalent ions at a range of plate separations.

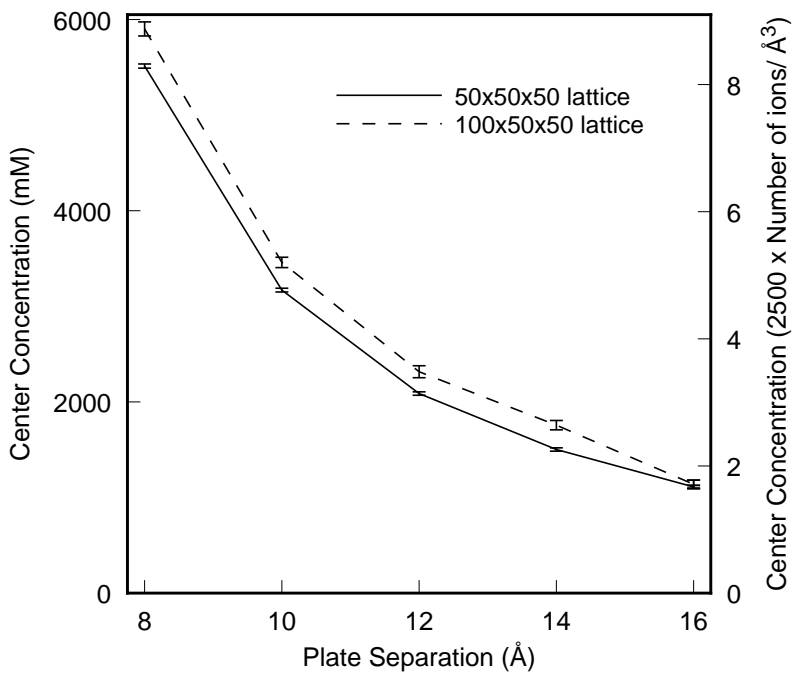

FIG. 3: Concentration of charges at the plane between the charges from simulations of univalent ions. Left axis has the same units as the pressure results. Right axis gives the number of charges on the center plane (50x50x50 lattice) or center two planes (100x50x50 lattice). This is one of the terms contributing to the osmotic pressure.

left axis of this plot gives the average number of ions in a $1 \AA$ by $50 \AA$ by $50 \AA$ rectangular box centered between the plates. Figure 4 shows the second term of Eq. 33 The differences in results from the two lattice sizes are modest, showing that the errors due to lattice discretization are small. Figure 5 shows the concentration profiles of the ions in solution from the simulations on the $50 \times 50 \times 50$ lattice. The ions are attracted to the plates, but a small density of ions remains in the center of the gap between the planes.

For the divalent ions we only consider the $50 \times 50 \times 50$ lattice. Here we use the heat-bath method for moving the particles and updating the electric fields. There are 10,000 equilibration steps and 200,000 measurement

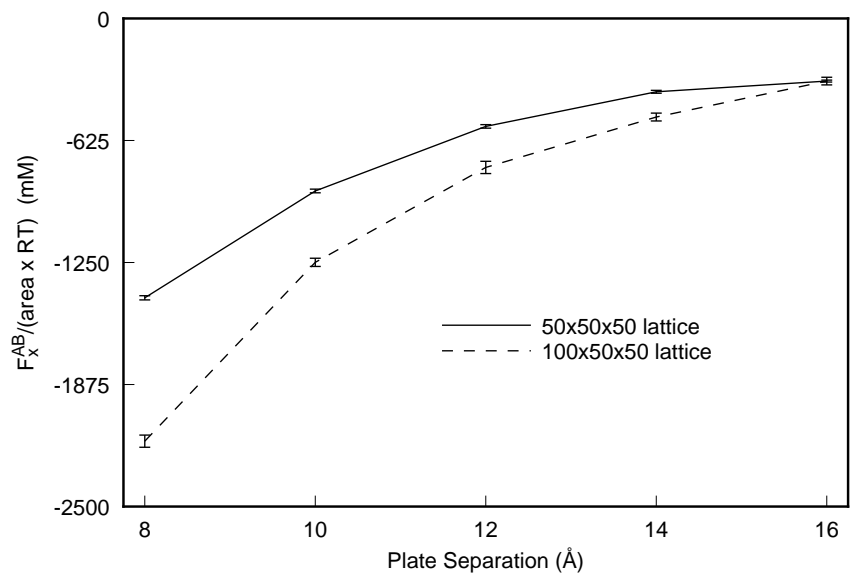

FIG. 4: Electrostatic force between the halves of the system for univalent ions. This is the second term contributing to the osmotic pressure.

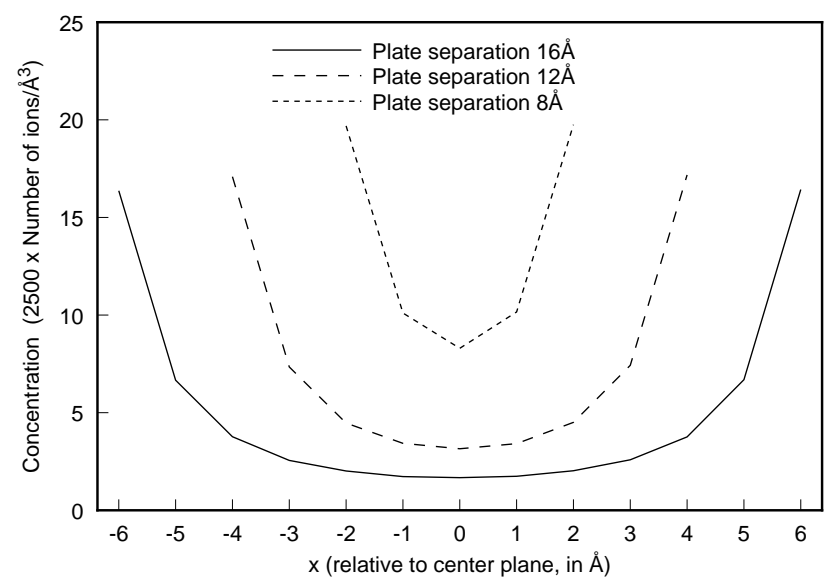

FIG. 5: Concentration profile of the univalent ions in solution for a range of plate separations. For each $x$, the average number of ions in the system a distance $x$ from the center plate is shown. The ion concentration is 0 outside of the range shown.

steps, and other parameters are the same as the univalent case. The solid line in Fig. [6 shows the pressure in the divalent case, while dashed line and dashed-dotted line show the first and second terms of Eq. 33. Qualitatively, our result agree with previous theoretical and Monte Carlo work, although a direct quantitative comparison is difficult because of the differences in how the interaction with the plate is treated. The concentration profiles of the ions in solution for these simulations are shown in Fig. 7 Note that the ions are much more tightly bound to the plates in the divalent case as compared with the univalent case.

The simulations discussed here would be difficult or impossible to perform with the simple particle Metropolis move, which does not adjust the field on neighboring plaquettes. By using the coupled Metropolis updating 


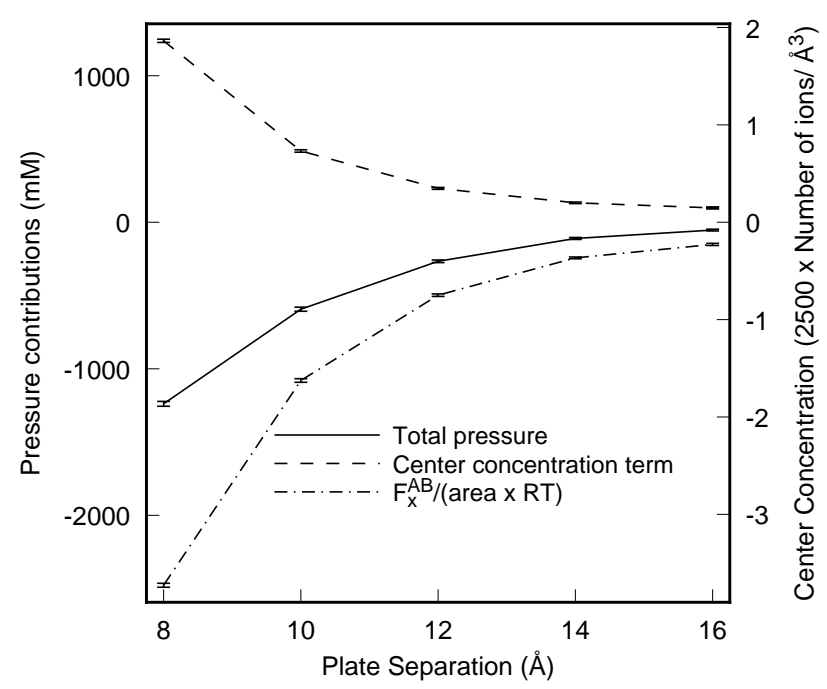

FIG. 6: Results from simulations on divalent ions at a range of plate separations on a 50x50x50 lattice. Shown with a solid line is the total osmotic pressure divided by $R T$. Shown with a dashed line and a dotted-dashed line are the two terms that contribute to it, the ion concentration on the center plane and the electrostatic force between the two halves of the system. The axis on the left gives the values in units of micromolars; the axis on the right gives the concentration on the center plane in terms of the number of ions in our system on the center plane.

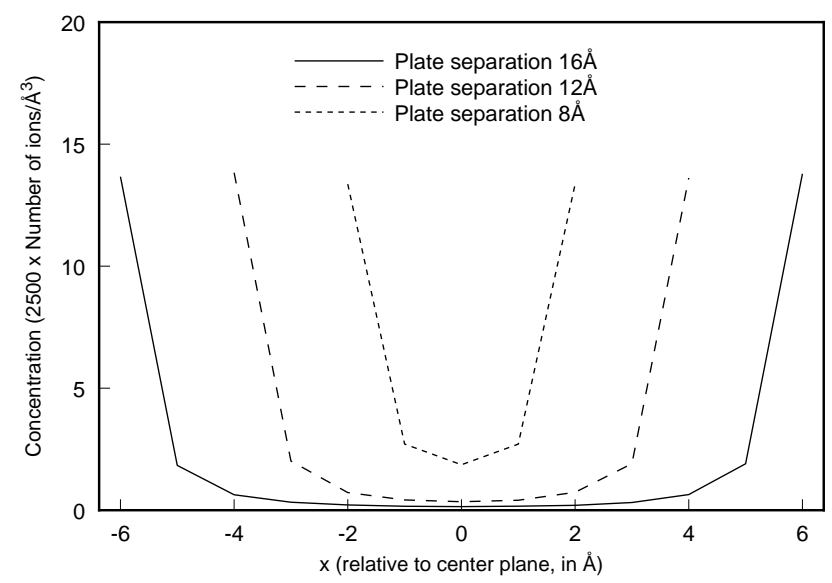

FIG. 7: Concentration profile of the divalent ions in solution for a range of plate separations. For each $x$, the average number of ions in the system a distance $x$ from the center plate is shown. The ion concentration is 0 outside of the range shown.

move described in this work we are able to increase the acceptance rate for particle moves in the univalent case to 0.13 from $10^{-4}$, and in the divalent case to 0.0056 from less than $10^{-6}$. The anisotropy of the $100 \times 50 \times 50$ lattice affects the acceptance rates. In this case the univalent acceptance rates using the coupled Metropolis move increases to 0.48 in the $x$ direction, but the acceptance rate

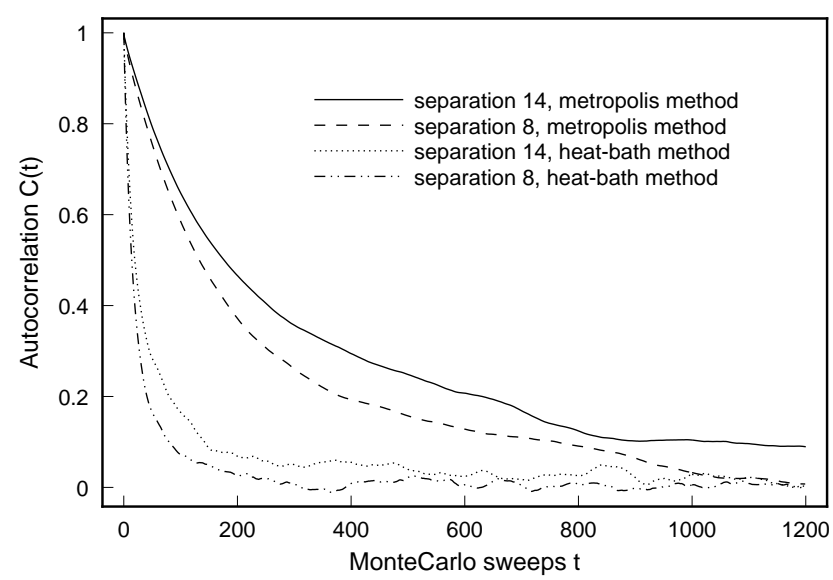

FIG. 8: Comparison of pressure autocorrelation functions using the coupled Metropolis move update, and the heat-bath particle move update. These autocorrelation functions are all from simulations with divalent ions.

for particle moves perpendicular to the $x$ direction drops to 0.0061 . This asymmetry occurs because the coupled update procedure is no longer able to effectively spread the change in electric field when a particle is moved in the $y$ or $z$ directions.

When using the heat-bath approach to moving the particles, the acceptance rate $(0.10$ for univalent ions and 0.0045 for divalent ions) is lower than the coupled Metropolis move acceptance rate, but still has a much greater acceptance rate than the simple Metropolis particle move that does not adjust the electric field on neighboring plaquettes. The advantage of the heat-bath approach is that it better decorrelates the system so that the observables have a shorter autocorrelation time. The autocorrelation function of a observable $A_{t}$ is given by

$$
C(t)=\frac{1}{N-t} \sum_{j=1}^{N-t}\left(A_{j}-\bar{A}\right)\left(A_{j+t}-\bar{A}\right),
$$

where $\bar{A}$ is the average of $A_{t}$. The autocorrelation function of the pressure is shown in Fig. 8 for plate separations of 8 and 14 with divalent ions using both the coupled Metropolis particle move update and the heatbath particle move update. Although the autocorrelation time (obtained by integrating the autocorrelation function) increases with the larger plate separation, the autocorrelation time with the heat-bath update is consistently smaller than the autocorrelation time for the coupled Metropolis update. Across all plate separations, the autocorrelation times from heat-bath updates were five to ten times smaller than the autocorrelation times from coupled Metropolis updates. This more than compensates for the additional computational cost per sweep (approximately twice that of the coupled Metropolis update) of the heat-bath update. 


\section{CONCLUSIONS}

The development of efficient local algorithms for Monte Carlo simulation of Coulomb systems with non-uniform dielectric constants is crucial for the study of the larger and more physically realistic biophysical systems of interest to researchers $9,10,11$. The technique of Maggs et al. shows great promise in fulfilling these goals, but it must be shown to be efficient and accurate in physically interesting parameter ranges. Studying a system of parallel plates screened by ion with a large dimensionless inverse temperature, we see that the simplest method of moving particles, where the electric field is only modi- fied on the link traversed by the particle, gives unusably small acceptance rates. By updating the electric field on plaquettes neighboring the traversed link, we can increase the acceptance rates to a usable level. Using a heat bath approach reduces the autocorrelation time of the simulation.

\section{ACKNOWLEDGMENTS}

The work of A. Duncan was supported in part by NSF grant PHY0244599. The work of R.D. Sedgewick and R.D. Coalson was supported by NSF grant CHE0092285.
[1] A.C. Maggs and V. Rossetto, Phys. Rev. Lett. 88, 196402 (2002)

[2] F. Allen et al., IBM Systems Journal 40, 310 (2001)

[3] L. Levrel, F. Alet, J. Rottler, and A.C. Maggs, Statphys22 Proceedings, to be published in PRAMANA [also available at cond-mat/0409350]

[4] R. Podgornik and B. Žekš, J. Chem. Soc., Faraday Trans. 2 84, 611 (1988)

[5] M.J. Stevens and M.O. Robbins, Europhys. Lett. 12, 81 (1990)

[6] L. Guldbrand, B. Jönsson, H. Wennerström, and P. Linse, J. Chem. Phys. 80, 2221 (1984)

[7] A.G. Moreira and R.R. Netz, Eur. Phys. J. E 8, 33 (2002)

[8] For a summary of these techniques see: A. Leach, "Molecular Modeling: Principles and Applications," Prentice Hall, 2001

[9] M. Zacharias, B.A. Luty, M.E. Davis and J.A. McCam- mon, J. Mol. Biol. 238, 455 (1994)

[10] D. Bashford and M. Karplus, Biochemistry 29, 10219 (1990)

[11] V. Spassov and D. Bashford, Protein Sci. 7, 2012 (1998)

[12] S.F. Edwards, Philos. Mag. 4, 1171 (1959);

[13] S.F. Edwards and A. Lenard, J. Math. Phys. 3, 778 (1962)

[14] R. Coalson and A. Duncan, J. Chem. Phys. 97, 5653 (1992)

[15] I. Montray and G. Münster, "Quantum Fields on a lattice," Cambridge Monographs on Mathematical Physics, 1997

[16] A.C. Maggs, J. Chem. Phys. 120, 3108 (2004)

[17] W.H. Press, B.P. Flannery, S.A. Teukolsky, and W.T. Vetterling, "Numerical Recipes in C: The Art of Scientific Computing," Cambridge University Press, 1992 